\title{
Developments in the Khintchine-Meinardus probabilistic method for asymptotic enumeration
}

\author{
Boris L. Granovsky \\ Department of Mathematics \\ Technion-Israel Institute of Technology \\ Haifa, 32000, Israel \\ mar18aa@techunix.technion.ac.il
}

\author{
Dudley Stark \\ School of Mathematical Sciences \\ Queen Mary, University of London \\ London E1 4NS, United Kingdom \\ D.S.Stark@maths .qmul .ac.uk
}

Submitted: Aug 7, 2014; Accepted: Nov 10, 2015; Published: Nov 27, 2015

Mathematics Subject Classifications: 05A16, 60F99, 81T25

\begin{abstract}
A theorem of Meinardus provides asymptotics of the number of weighted partitions under certain assumptions on associated ordinary and Dirichlet generating functions. The ordinary generating functions are closely related to Euler's generating function $\prod_{k=1}^{\infty} S\left(z^{k}\right)$ for partitions, where $S(z)=(1-z)^{-1}$. By applying a method due to Khintchine, we extend Meinardus' theorem to find the asymptotics of the Taylor coefficients of generating functions of the form $\prod_{k=1}^{\infty} S\left(a_{k} z^{k}\right)^{b_{k}}$ for sequences $a_{k}, b_{k}$ and general $S(z)$. We also reformulate the hypotheses of the theorem in terms of the above generating functions. This allows novel applications of the method. In particular, we prove rigorously the asymptotics of Gentile statistics and derive the asymptotics of combinatorial objects with distinct components.
\end{abstract}

Keywords: Meinardus' theorem; asymptotic enumeration; Dirichlet generating functions; models of ideal gas and of quantum field theory

\section{Introduction}

Meinardus [11] proved a theorem about the asymptotics of weighted partitions with weights satisfying certain conditions. His result was extended to the combinatorial objects called assemblies and selections in [5] and to Dirichlet generating functions for weights, with multiple singularities in [7]. In this paper, we extend Meinardus' theorem further to a general framework, which encompasses a variety of models in physics and combinatorics, including previous results.

Let $f$ be a generating function of a nonnegative sequence $\left\{c_{n}, n \geqslant 0, c_{0}=1\right\}$ :

$$
f(z)=\sum_{n \geqslant 0} c_{n} z^{n}
$$


with radius of convergence 1 . As an example, consider the number of weighted partitions $c_{n}$ of size $n$, determined by the generating function identity

$$
\sum_{n=0}^{\infty} c_{n} z^{n}=\prod_{k=1}^{\infty}\left(1-z^{k}\right)^{-b_{k}}, \quad|z|<1,
$$

for some sequence of real numbers $b_{k} \geqslant 0, k \geqslant 1$. If $b_{k}=1$ for all $k \geqslant 1$, then $c_{n}$ is the number of integer partitions. Meinardus [11] proved a theorem giving the asymptotics of $c_{n}$ under certain assumptions on the sequence $\left\{b_{k}\right\}$.

The generating function in (2) may be expressed as $\prod_{k=1}^{\infty}\left(S\left(z^{k}\right)\right)^{b_{k}}$, where $S(z)=$ $(1-z)^{-1}$. This observation allows the following natural generalization. Let $f$ in (1) be of the form:

$$
f(z)=\prod_{k=1}^{\infty}\left(S\left(a_{k} z^{k}\right)\right)^{b_{k}},
$$

with given sequences $0<a_{k} \leqslant 1, b_{k} \geqslant 0, k \geqslant 1$, and a given function $S(z)$.

This is a particular case of the class of general multiplicative models, introduced and studied by Vershik ([16]). In the setting (3), in the case of weighted partitions, a combinatorial meaning can be attributed to the parameters $a_{k}, b_{k}$. Namely, if $b_{k}=1$, then $a_{k}$ can be viewed as a properly scaled number of colours for each component of size $k$, such that given $l$ components of size $k$, the total number of colourings is $a_{k}^{l}$. Equivalently, to each particular partition of $n$, say $n=\sum_{l=1}^{n} j_{l} k_{l}$, is prescribed the weight $\prod_{l=1}^{n} a_{k_{l}}^{j_{l}}$. On the other hand, if $a_{k}=1$, then given $l$ components of size $k$, the total number of colourings equals the number of distributions of $b_{k}$ indistinguishable balls among $l$ cells, so that in this model $b_{k}$ has a meaning of a scaled number of types prescribed to a component of size $k$.

Yakubovich ([18]) derived the limit shapes for models (3) in the case $a_{k}=1, k \geqslant$ 1, under some analytical conditions on $S$ and $b_{k}$. Note that past versions [5]-[7] of Meinardus' theorem deal with the asymptotics of $c_{n}, n \rightarrow \infty$, when $a_{k}=1, k \geqslant 1$, for three cases of the function $S$, corresponding to the three classic models of ideal gas in statistical mechanics, namely, Maxwell-Boltzmann, Bose-Einstein and Fermi-Dirac. They are mathematically equivalent to aforementioned combinatorial models: assemblies, weighted partitions and selections, respectively. In this context, $n$ is a total energy of a system, which is partitioned into integer energy levels $k_{1}, \ldots, k_{n}$, so that $n=j_{1} k_{1}+\ldots+$ $j_{n} k_{n}$, where $j_{1}, \ldots, j_{n}$ are occupation numbers which are equal to the numbers of particles at corresponding energy levels.

Our objective in this paper is to derive the asymptotics $c_{n}, n \rightarrow \infty$, in the general framework (3). We suppose that $S(0)=1$ and that $S(z)$ can be expanded in a power series with radius of convergence $\geqslant 1$ and non-negative coefficients $d_{j}$ :

$$
S(z)=\sum_{j=0}^{\infty} d_{j} z^{j}
$$


with $d_{0}=1$, and that $\log S(z)$ can be expanded as

$$
\log S(z)=\sum_{j=1}^{\infty} \xi_{j} z^{j}
$$

with radius of convergence 1 . We note that the assumption $d_{j} \geqslant 0, j \geqslant 0$ is necessary for the implementation of the Khintchine probabilistic approach (see (19),(21) below).

From (3) and (5) one can express the coefficients $\Lambda_{k}$ of the power series for the function $\log f(z)$, with radius of convergence 1 :

$$
\log f(z)=\sum_{k=1}^{\infty} \Lambda_{k} z^{k}, \quad \Lambda_{k}=\sum_{j \mid k} b_{j} a_{j}^{k / j} \xi_{k / j} .
$$

We define the Dirichlet generating function for the sequence $\Lambda_{k}$ :

$$
D(s)=\sum_{k=1}^{\infty} \Lambda_{k} k^{-s}
$$

which by virtue of (6) admits the following representation

$$
D(s)=\sum_{k=1}^{\infty} \sum_{j=1}^{\infty} b_{k} \xi_{j} a_{k}^{j}(j k)^{-s}
$$

as long as $\Re(s)$ is large enough so that the double Dirichlet series in (8) converges absolutely. If $a_{k}=a, 0<a \leqslant 1$ for all $k \geqslant 1$, then $D(s)$ can be factored as

$$
D(s)=D_{b}(s) D_{\xi, a}(s),
$$

where

$$
D_{\xi, a}(s)=\sum_{j=1}^{\infty} a^{j} \xi_{j} j^{-s}
$$

and

$$
D_{b}(s)=\sum_{k=1}^{\infty} b_{k} k^{-s} .
$$

The greater generality of (3) than in previous versions of Meinardus' theorem will allow novel applications. The proof of Theorem 1, stated below, is a substantial modification of the method used in $[5,6,7]$.

We suppose that $\Lambda_{k}$ and $D(s)$ satisfy conditions $(I)-(I I I)$, which are modifications of the three original Meinardus' conditions in [11].

Condition $(\boldsymbol{I})$. The Dirichlet generating function $D(s), s=\sigma+i t$ is analytic in the half-plane $\sigma>\rho_{r}>0$ and it has $r \geqslant 1$ simple poles at positions $0<\rho_{1}<\rho_{2}<\ldots<\rho_{r}$, with positive residues $A_{1}, A_{2}, \ldots, A_{r}$ respectively. It may also happen that $D(s)$ has a 
simple pole at 0 with residue $A_{0}$. (If $D(s)$ is analytic at 0 , we take $A_{0}=0$ ). Moreover, there is a constant $0<C_{0} \leqslant 1$, such that the function $D(s), s=\sigma+i t$, has a meromorphic continuation to the half-plane

$$
\mathcal{H}=\left\{s: \sigma \geqslant-C_{0}\right\}
$$

on which it is analytic except for the above $r$ or $r+1$ simple poles.

Condition $(\boldsymbol{I I})$. There is a constant $C_{1}>0$ such that

$$
D(s)=O\left(|t|^{C_{1}}\right), \quad t \rightarrow \infty
$$

uniformly for $s=\sigma+i t \in \mathcal{H}$.

Condition (III).

The following property of the parameters $a_{k}, b_{k}$ holds:

$$
b_{k} a_{k}^{l_{0}} \geqslant C_{2} k^{\rho_{r}-1}, k \geqslant 1, C_{2}>0,
$$

where

$$
l_{0}:=\min \left\{j>0: d_{j}>0\right\} .
$$

Moreover, if $l_{0}>1$ then for some fixed $\epsilon>0$ and for small enough $\delta$,

$$
2 \sum_{k=1}^{\infty} \Lambda_{k} e^{-k \delta} \sin ^{2}(\pi k \alpha) \geqslant\left(1+\frac{\rho_{r}}{2}+\epsilon\right)|\log \delta|, \quad\left(2 l_{0}\right)^{-1} \leqslant|\alpha| \leqslant 1 / 2, \quad l_{0}>1,
$$

where $\Lambda_{k}$ is as defined in (6).

In order to state our main result, we need some more notations, which were also used in [7]. Define the finite set

$$
\tilde{\Upsilon}_{r}=\left\{\sum_{k=0}^{r-1} \tilde{d}_{k}\left(\rho_{r}-\rho_{k}\right): \tilde{d}_{k} \in \mathbb{Z}_{+}, \sum_{k=0}^{r-1} \tilde{d}_{k} \geqslant 2\right\} \cap\left(0, \rho_{r}+1\right], \quad r \geqslant 1,
$$

where we have set $\rho_{0}=0$ and let $\mathbb{Z}_{+}$denote the set of nonnegative integers. Let $0<$ $\alpha_{1}<\alpha_{2}<\cdots<\alpha_{\left|\tilde{\Upsilon}_{r}\right|} \leqslant \rho_{r}+1$ be all ordered numbers forming the set $\tilde{\Upsilon}_{r}$. Clearly, $\alpha_{1}=2\left(\rho_{r}-\rho_{r-1}\right)$, if the set $\tilde{\Upsilon}_{r}$ is not empty. We also define the finite set

$$
\Upsilon_{r}=\tilde{\Upsilon}_{r} \cup\left\{\rho_{r}-\rho_{k}: k=0,1, \ldots, r-1\right\},
$$

observing that some of the differences $\rho_{r}-\rho_{k}, k=0, \ldots, r-1$ may fall into the set $\tilde{\Upsilon}_{r}$. We let $0<\lambda_{1}<\lambda_{2}<\cdots<\lambda_{\left|\Upsilon_{r}\right|}$ be all ordered numbers forming the set $\Upsilon_{r}$.

Theorem 1 Suppose conditions $(I)-(I I I)$ are satisfied.

Suppose that $c_{n}$ has ordinary generating function of the form (3), where $0<a_{k} \leqslant 1$ and $b_{k} \geqslant 0, k \geqslant 1$, that (11) in Condition (III) is satisfied for a constant $C_{2}>0$, and that

$$
\frac{d^{2}}{d \delta^{2}} \log S\left(e^{-\delta}\right)>0, \quad \delta>0
$$


We then have, as $n \rightarrow \infty$,

$$
c_{n} \sim H n^{-\frac{2+\rho_{r}-2 A_{0}}{2\left(\rho_{r}+1\right)}} \exp \left(\sum_{l=0}^{r} P_{l} n^{\frac{\rho_{l}}{\rho_{r}+1}}+\sum_{l=0}^{r} \hat{h}_{l} \sum_{s: \lambda_{s} \leqslant \rho_{l}} K_{s, l} n^{\frac{\rho_{l}-\lambda_{s}}{\rho_{r}+1}}\right),
$$

where $H, P_{l}, \hat{h}_{l}$ and $K_{s, l}$ are constants. In particular, if $r=1$, then $K_{s, l}=0$ for all $s$ and $l$,

$$
P_{1}=\left(1+\frac{1}{\rho_{1}}\right)\left(A_{1} \Gamma\left(\rho_{1}+1\right)\right)^{1 /\left(\rho_{1}+1\right)}
$$

and

$$
H=e^{\Theta-\gamma A_{0}}\left(2 \pi\left(1+\rho_{1}\right)\right)^{-1 / 2}\left(A_{1} \Gamma\left(\rho_{1}+1\right)\right)^{\frac{1-2 A_{0}}{2\left(\rho_{1}+1\right)}},
$$

where

$$
\Theta:=\lim _{s \rightarrow 0}\left(D(s)-A_{0} s^{-1}\right)
$$

and $\gamma$ is Euler's constant.

Remark The sums in (17) could be taken from $l=1$ to $r$ and $P_{0}$ and $\hat{h}_{0} K_{0,0}$ absorbed into $H$, but we prefer not to do that as the constants in (17) arise naturally from the proof of Theorem 1 . The constants $P_{l}, K_{s, l}$ in (17) are calculated by the recursive method of [7]. We do not repeat the description of the method here.

Theorem 1 generalizes the seminal results by Khintchine [10] and Meinardus [11], as well as their extensions in $[5,7]$, and implies the results therein, including expansive weighted partitions, for which $S(z)=(1-z)^{-1}, a_{k}=1, k \geqslant 1$ and $b_{k}=k^{r-1}, k \geqslant 1$ for some $r>0$.

Example This example shows that (11) is not implied by the other hypotheses of Theorem 1. Let $a_{k}=1$ for all $k$, let $b_{k}=k^{\rho_{1}-1}$ and let $\xi_{k}=k^{\rho_{2}-1}$, where $0<\rho_{1}<\rho_{2}$. Then, $D_{\xi, 1}(s)=\zeta\left(s+1-\rho_{2}\right), D_{b}(s)=\zeta\left(s+1-\rho_{1}\right)$, where $\zeta$ is the Riemann zeta function, and $D(s)=D_{b}(s) D_{\xi, 1}(s)$ has simple poles at $\rho_{1}$ and $\rho_{2}$. Moreover, $S(z)=$ $\exp \left(\sum_{k=1}^{\infty} k^{\rho_{2}-1} z^{k}\right)$ has radius of convergence 1 and it is easy to check that (16) is satisfied. However,(11) is violated.

As in [5]-[7], the proof of Theorem 1 is based on the Khintchine type representation $[10]$

$$
c_{n}=e^{n \delta} f_{n}\left(e^{-\delta}\right) \mathbb{P}\left(U_{n}=n\right), \quad n \geqslant 1,
$$

where $\delta>0$ is a free parameter,

$$
f_{n}(z)=\prod_{k=1}^{n} S\left(a_{k} z^{k}\right)^{b_{k}}
$$

is the $n$-truncation of $f$ in $(3)$, and $U_{n}, n \geqslant 1$ are integer-valued random variables with characteristic functions defined by

$$
\phi_{n}(\alpha)=\mathbb{E}\left(e^{2 \pi i \alpha U_{n}}\right)=\prod_{k=1}^{n}\left(\frac{S\left(a_{k} e^{2 \pi i k \alpha-k \delta}\right)}{S\left(a_{k} e^{-k \delta}\right)}\right)^{b_{k}}, \quad n \geqslant 1, \quad \alpha \in \mathbb{R} .
$$


Khintchine established (19) for the three basic models of statistical mechanics. For general multiplicative measures (19) was stated in equation (4) of [5]. It remains to analyze each of the three factors of the right hand side of (19).

The proof of Theorem 1 is similar in form to proofs in $[5,6,7]$, however there are notable differences. The form of the generating function $f(z)$ in $(3)$ is much more general than in the cases of the aforementioned classic combinatorial structures $(=$ models of statistical mechanics). Also recall that in the previous papers, the parameters $a_{k}$ in (3) were always taken to be equal 1 . However, we still have a nice representation of $D(s)$ given in (9), which allows us to proceed with the Meinardus-Khintchine method. The basic method of proof is an analysis of the three factors of (19) when $\delta=\delta_{n}$ is chosen to be the solution of the equation $\mathbb{E} U_{n}=n, n \geqslant 1$. The convexity assumption (16) is made in order to guarantee that there exists a unique solution $\delta_{n}$ whose asymptotics we may obtain. Regarding conditions $(I),(I I)$, and $(I I I)$, conditions $(I)$ and $(I I)$ are similar to the corresponding conditions in [7], in which Dirichlet generating functions with multiple poles were considered. An asymptotic equation for $\delta_{n}$ is obtained from condition $(I)$ and $(I I)$ by using Mellin transforms and then changing the contour of integration. Assumption (13) of Condition (III) is stronger than the original condition of Meinardus, however here it is used to prove a local limit theorem, the proof of which requires, in addition, assumption (11), which involves both $a_{k}$ and $b_{k}$.

The proof of Theorem 1 is contained in Appendix A.

\section{Two historical remarks}

1. Khintchine's probabilistic approach for asymptotic enumeration resulted in the representation (19). Explaining his idea to replace the saddle point method with a local limit theorem, Khintchine wrote in [10]:" ... the main novelty of this approach consists of replacing the complicated analytical apparatus (the method of Darwin-Fowler) by ... the well developed limit theorems of the theory of probability ... that can form the analytical basis for all the computational formulas of statistical mechanics." In [5], the practical advantages of this idea regarding the asymptotic problems considered was explained.

2. The Khintchine-Meinardus method used in this work covers a variety of models given by generating functions $\mathcal{F}(\delta)=f\left(e^{-\delta}\right)$ exhibiting exponential asymptotics, as $\delta \rightarrow$ $0^{+}$(see Lemma 1 below) which are essentially implied by Meinardus Condition 1 of the main theorem. In this connection note that there exists a rich literature (see e.g. [2]) devoted to the case of moderately, i.e. non exponentially, growing $\mathcal{F}(\delta), \delta \rightarrow 0$. Such models are studied by a quite different singularity analysis.

\section{Gentile statistics}

Gentile statistics is a model studied in physics $[4,13,15]$, which counts partitions of an integer $n$ with no part occurring more than $\eta-1$ times, where $\eta \geqslant 2$ is a parameter. When $\eta=2$, Fermi-Dirac statistics are obtained and when $\eta=\infty$, Bose-Einstein statistics, with uniform weights $b_{k}=1, k \geqslant 1$ result. As far as we know, no rigorous derivation of the 
asymptotics of Gentile statistics has previously been given, although Theorem 2 below was anticipated in approximation (23) of [13]. In this work we derive the aforementioned theorem as a special case of our Theorem 1.

Gentile statistics are Taylor coefficients of the generating function

$$
f(z)=\prod_{k=1}^{\infty} \frac{1-z^{\eta k}}{1-z^{k}}, \quad|z|<1, \quad \eta \geqslant 2 \text { is an integer. }
$$

We remark that there is another natural interpretation of the Gentile statistics, which is the number of integer partitions with no part size divisible by $\eta$, where part sizes can appear an unlimited number of times. Gentile statistics fit into the framework (3) of Theorem 1 with

$$
S(z)=\frac{1-z^{\eta}}{1-z}, \quad|z|<1, \quad \eta \geqslant 2 \text { is an integer }
$$

and $a_{k}=b_{k}=1, \quad k \geqslant 1$.

Theorem 2 Gentile statistics have asymptotics

$$
c_{n} \sim \sqrt{\frac{\kappa}{4 \pi \eta}} n^{-3 / 4} e^{2 \kappa \sqrt{n}}
$$

where

$$
\kappa=\sqrt{\zeta(2)\left(1-\eta^{-1}\right)}, \quad \eta \geqslant 2 \text { is an integer. }
$$

Proof We will show that all the conditions of Theorem 1 are satisfied for Gentile statistics. In order to show that (16) holds for $\eta>1$, we calculate

$$
\frac{d^{2}}{d \delta^{2}} \log S\left(e^{-\delta}\right)=\frac{e^{\delta}}{\left(e^{\delta}-1\right)^{2}}-\frac{\eta^{2} e^{\eta \delta}}{\left(e^{\eta \delta}-1\right)^{2}} .
$$

We have

$$
\begin{aligned}
\frac{d}{d \eta} \frac{\eta^{2} e^{\eta \delta}}{\left(e^{\eta \delta}-1\right)^{2}} & =\frac{\eta e^{\eta \delta}\left[e^{\eta \delta}(2-\delta \eta)-(2+\delta \eta)\right]}{\left(e^{\eta \delta}-1\right)^{3}} \\
& =\frac{\eta e^{\eta \delta} g(\eta \delta)}{\left(e^{\eta \delta}-1\right)^{3}},
\end{aligned}
$$

where $g(x)=e^{x}(2-x)-(2+x)$. Taking the derivative of $g$ produces $g^{\prime}(x)=e^{x}(1-x)-1<$ 0 for $x>0$, which, together with $g(0)=0$, implies that $g(x)<0$ for $x>0$. Combining this with the fact that $\frac{d^{2}}{d \delta^{2}} \log S\left(e^{-\delta}\right)=0$, if $\eta=1$, we conclude that (16) holds, for all $\eta>1$.

It remains to be shown that conditions $(I)-(I I I)$ are satisfied for the model considered. We have

$$
\log f(z)=\sum_{k=1}^{\infty} \sum_{j=1}^{\infty}\left(\frac{z^{j k}}{j}-\frac{z^{j k \eta}}{j}\right), \quad|z|<1,
$$


and so, by (6), (7) and (8),

$$
\begin{aligned}
D(s) & =\sum_{k=1}^{\infty} \sum_{j=1}^{\infty}\left(\frac{(j k)^{-s}}{j}-\frac{(j k \eta)^{-s}}{j}\right) \\
& =\zeta(s) \zeta(s+1)\left(1-\eta^{-s}\right) .
\end{aligned}
$$

Conditions $(I)$ and $(I I)$ are satisfied with any $0<C_{0}<1$ because of the analytic continuation of the Riemann zeta function and the well known bound

$$
\zeta(x+i y)=O\left(|y|^{C}\right), \quad y \rightarrow \infty,
$$

for a constant $C>0$, uniformly in $x$. It is easy to check that $l_{0}=1$ and $b_{k} a_{k}=1=k^{\rho_{1}-1}$, where $\rho_{1}=1$, and so (11) is satisfied. Hence condition $(I I I)$ is satisfied. Moreover,

$$
\begin{gathered}
r=1, \rho_{0}=0, \rho_{1}=1, A_{0}=\lim _{s \rightarrow 0} s D(s)=0, A_{1}=\zeta(2)\left(1-\eta^{-1}\right), \\
\Theta=\lim _{s \rightarrow 0} D(s)=\zeta(0) \log \eta .
\end{gathered}
$$

By the argument preceding Proposition 1 in Appendix A, this says that the integrand $\delta_{n}^{-s} \Gamma(s) D(s)$ has a simple pole at $s=0$ with residue $\Theta=\zeta(0) \log \eta$ and a simple pole at $s=1$ with residue $\zeta(2)\left(1-\eta^{-1}\right) \delta_{n}^{-1}$. As a result, in the case considered $\delta_{n}=\hat{h}_{1}^{1 / 2} n^{-1 / 2}-$

$2^{-1} \hat{h}_{0} n^{-1}+O\left(n^{-\frac{C_{0}}{2}-1}\right)$, where $\hat{h}_{1}$ and $\hat{h}_{0}$ are defined by (35) and (36), and we arrive at the claimed asymptotic formula for $c_{n}$.

\section{Asymptotic enumeration for distinct part sizes}

Weighted partitions fit our framework (3) with $S(z)=(1-z)^{-1}, a_{k}=1, k \geqslant 1$ and weights $b_{k}$. When $b_{k}=1, k \geqslant 1$, Theorem 1 gives the principal term in the asymptotical expansion of the number of partitions of $n$, obtained by Hardy and Ramanujan in their famous paper [9]. If $S(z)=1+z, a_{k}=1, b_{k}=k^{r-1}, r>0, k \geqslant 1$, then $c_{n}$ enumerates weighted partitions having no repeated parts, called expansive selections. The asymptotics of expansive selections were also studied in [6].

In this section, we find the asymptotics of $c_{n}$ induced by the generating function

$$
f(z)=\sum_{n=0}^{\infty} c_{n} z^{n}=\prod_{k=1}^{\infty}\left(1+k^{-q} z^{k}\right),|z|<1, \quad q>0 .
$$

The model fits the setting (3) with $S(z)=1+z, b_{k}=1, a_{k}=k^{-q}, k \geqslant 1$ and it can be considered as a colored selection with parameter $k^{-q}$ proportional to the number $m_{k}$ of colors of a component of size $k$, e.g. $m_{k}=y^{k} k^{-q}$, for some $y>1$. A particular case of the model, when $q=1$ was studied in Section 4.1 .6 of [8] where it was proven, with the help of a Tauberian theorem, that in this case

$$
\lim _{n \rightarrow \infty} c_{n}=e^{-\gamma}
$$


and it was established the rate of convergence of $c_{n}, n \rightarrow \infty$. Also, in [8] it was shown that $c_{n}$ is equal to the probability that a random polynomial of order $n$ is a product of irreducible factors of different degrees. In [12], Section 11, it was demonstrated that $c_{n}$ can be treated as the probability that a random permutation on $n$ letters has distinct cycle lengths, and another proof of (24) was suggested. The generating function (23) is discussed in [2] and the method therein applied when $q>1$ to give the rate of decay $n^{-q}$ of $c_{n}$, up to an unspecified constant. By elementary techniques we show below that for $q>1, c_{n} \sim W(q) n^{-q}$, where $W(q):=\sum_{n=0}^{\infty} c_{n}$. In Appendix B, an expression for the constant $W(q)$ as an infinite product is derived.

Finally, note that in [12], (11.35), it was shown that for $q=2$, the generating function $f(z)$ can not be analytically continued beyond the unit circle.

Theorem 3 Let

$$
\sum_{n=0}^{\infty} c_{n} z^{n}=\prod_{k=1}^{\infty}\left(1+k^{-q} z^{k}\right),|z|<1 .
$$

If $0<q<1$, then $c_{n}$ has asymptotics given by (17) with $r=\max \{j \geqslant 1: 1-q j>0\}$ and $\rho_{l}=1-q l, l=1, \ldots$. If $q>1$, then, for a constant $W(q)>0$ depending only on $q$,

$$
c_{n} \sim W(q) n^{-q}
$$

as $n \rightarrow \infty$.

\section{Proof}

The case $0<q<1$.

We will apply Theorem 1. Assumption (16) is easy to verify. We have

$$
\log f(z)=\sum_{k \geqslant 1} \log \left(1+\frac{z^{k}}{k^{q}}\right)=\sum_{k \geqslant 1} \sum_{j \geqslant 1}(-1)^{j-1} \frac{z^{k j}}{j k^{q j}}, \quad|z|<1
$$

and so, by (6) and (8),

$$
D(s)=D(s ; q)=\sum_{k \geqslant 1} \sum_{j \geqslant 1}(-1)^{j-1} \frac{(k j)^{-s}}{j k^{q j}}=\sum_{k \geqslant 1} \sum_{j \geqslant 1} \frac{(-1)^{j-1}}{j^{s+1} k^{s+q j}} .
$$

We claim that the function $D(s ; q)$ allows analytic continuation to the set $\mathbb{C}$ except for the poles in $H_{q}:=\{s=1-q j, j=1,2, \ldots, q<1\}$. Changing the order of summation, we write

$$
D(s ; q)=\sum_{j \geqslant 1} \sum_{k \geqslant 1} \frac{(-1)^{j-1}}{j^{s+1} k^{s+q j}}=\sum_{j \geqslant 1} \frac{(-1)^{j-1}}{j^{s+1}} \zeta(s+q j), \quad \Re(s)>0, s \notin H_{q} .
$$

Note that

$$
\zeta(s+q j)=1+\sum_{n=2}^{\infty} \frac{1}{n^{s+q j}}:=1+\Phi(s ; q),
$$


where the function $\Phi(s ; q)$ is analytic for $s \in \mathbb{C} \backslash H_{q}$, and moreover

$$
\Phi(s ; q)=O\left(2^{-q j}\right), \quad j \rightarrow \infty, \quad q>0,
$$

uniformly in $s$ from any compact subset of $\mathbb{C} \backslash H_{q}$. This implies that the series

$$
\sum_{j \geqslant 1} \frac{(-1)^{j-1}}{j^{s+1}} \Phi(s ; q)
$$

converges absolutely and uniformly on any compact subset of $\mathbb{C} \backslash H_{q}$. By the Weierstrass convergence theorem, this implies that the series above is analytic in the above indicated domain. Since the function

$$
\sum_{j \geqslant 1} \frac{(-1)^{j-1}}{j^{s+1}}=-\left(2^{-s}-1\right) \zeta(s+1)
$$

is analytic in $\mathbb{C}$, our claim is proven. This allows us to conclude that condition $I$ of Theorem 1 holds with $r=\max \{j \geqslant 1: 1-q j>0\}$ simple positive poles at $\rho_{l}=$ $1-q(r-l+1), l=1, \ldots r$ and with $0<C_{0}<1$ defined by

$$
C_{0}= \begin{cases}(r+1) q-1-\epsilon, \quad 0<\epsilon<(r+1) q-1, & \text { if }(r+1) q \leqslant 2 \\ \text { any number in }(0,1), & \text { if }(r+1) q>2 .\end{cases}
$$

Condition (II) follows from (22) and (26). Finally, $l_{0}=1$ in the case considered because $S(z)=1+z$ and $b_{k} a_{k}=k^{-q}=k^{\rho_{r}-1}$ and so (11) is satisfied. Hence condition $(I I I)$ is satisfied, by Lemma 1 in [5] which states that the bound (11) on $b_{k}$ supplies (13).

The poles $\rho_{l}=1-q l$ are such that their differences are multiples of $q$ and so $\Upsilon_{r}$ defined by (15) will contain the multiples of $q$ in $\left(0, \rho_{r}+1\right]$. However, the set $\tilde{\Upsilon}_{r}$ defined by (14) may contain additional elements. For example, taking $\tilde{d}_{0}=2$ and all other $\tilde{d}_{k}=0$ in (15) gives $2 \rho_{r}=2(1-q)<2-q=\rho_{r}+1$ and so $2 \rho_{r} \in \tilde{\Upsilon}_{r}$. It will be the case that $3 \rho_{r} \in \tilde{\Upsilon}_{r}$ if and only if $q>1 / 2$. The set $\Upsilon_{r}$ defined by (15) will also contain $\rho_{r}$ (taking $k=0)$. Thus, the $\lambda_{s}$ will contain the multiples of $q$ and of $\rho_{r}=1-q$ in $\left(0, \rho_{r}+1\right]$. The coefficients $K_{s, l}$ in (17) are implicitly given by the recursion

$$
n \delta_{n}^{\rho_{r}+1}-\sum_{k=0}^{r} \hat{h}_{k} \delta_{n}^{\rho_{r}-\rho_{k}}=o\left(n^{-1}\right)
$$

which comes from Proposition 1 of [7]. Note that the definition of $\Upsilon_{r}$ is motivated by the solution of the above recursion. To find the $K_{s, l}$ in examples such as this one, for which $\delta_{n}$ has a series expansion, one would probably want to find the asymptotic solution of the recursion above by computer.

\section{The case $q>1$.}

Theorem 1 is not applicable in this case, because all poles $1-q j, j \geqslant 1, \quad q>1$ of the function $D(s ; q)$ in $(26)$, are negative. From

$$
f(z)=\prod_{k=1}^{\infty}\left(1+z^{k} k^{-q}\right)=\sum_{n=0}^{\infty} c_{n} z^{n}, \quad|z| \leqslant 1, c_{0}=1 \quad q>1
$$


we have

$$
f(1)=\prod_{k=1}^{\infty}\left(1+k^{-q}\right):=W(q)<\infty, \quad q>1,
$$

since the convergence of the infinite product in (28) is equivalent to the convergence of the series

$$
\sum_{k=1}^{\infty} k^{-q}<\infty, \quad q>1
$$

By (27) and (28),

$$
W(q)=\sum_{n=0}^{\infty} c_{n}, q>1 .
$$

In proving that $\lim _{n \rightarrow \infty} n^{q} c_{n}=W(q)$, we define

$$
f_{l}(z):=\prod_{k=1}^{l}\left(1+z^{k} k^{-q}\right)=\sum_{k=0}^{l(l+1) / 2} c_{k}^{(l)} z^{k} .
$$

Two key facts follow from the definitions of $f$ and $f_{l}$ :

$$
0 \leqslant c_{k}^{(l)} \leqslant c_{k}, 1 \leqslant l<k ; \quad c_{k}^{(l)}=c_{k}, l \geqslant k, k=0,1, \ldots l,
$$

Also note that $c_{k}^{(l)}$ counts the number of distinct partitions of $k$ with parts at most $l$ and that

$$
c_{k}^{(n-k-1)}=0, \text { if } k>\frac{(n-k-1)(n-k)}{2},
$$

because $f_{l}(z)$ is a polynomial in $z$ of degree $\frac{l(l+1)}{2}$. From (27) we obtain the recurrence relation

$$
\begin{aligned}
c_{n}= & n^{-q} c_{0}+(n-1)^{-q} c_{1}+\cdots+\left(n-n^{*}\right)^{-q} c_{n^{*}} \\
& +\left(n-n^{*}-1\right)^{-q} c_{\left(n^{*}+1\right)}^{\left(n-n^{*}-2\right)}+\cdots++2^{-q} c_{n-2}^{(1)}+c_{n-1}^{(0)}, \quad n \geqslant 2,
\end{aligned}
$$

where, by virtue of the second fact in (30),

$$
n^{*}= \begin{cases}{\left[\frac{n}{2}\right],} & \text { if } \mathrm{n} \text { is odd } \\ {\left[\frac{n}{2}\right]-1,} & \text { if } \mathrm{n} \text { is even }\end{cases}
$$

The condition (31) is equivalent to $n-1 \geqslant k>k_{n}^{*}:=n+\frac{1}{2}-\frac{1}{2} \sqrt{8 n+1}$ and it expresses the fact that dictinct partitions of $k_{n}^{*} \sim n-\sqrt{2 n}, n \rightarrow \infty$ with largest part at most $n-k_{n}^{*} \sim \sqrt{2 n}, n \rightarrow \infty$ do not exist. Hence, (32) can be written as

$$
n^{q} c_{n}=\sum_{k=0}^{n^{\epsilon}}\left(\frac{n-k}{n}\right)^{-q} c_{k}+\sum_{k=n^{\epsilon}+1}^{n^{*}}\left(\frac{n-k}{n}\right)^{-q} c_{k}+\sum_{k=n^{*}+1}^{k_{n}^{*}}\left(\frac{n-k}{n}\right)^{-q} c_{k}^{(n-k-1)}
$$


for some $\epsilon>0$. The expression (33) and the first fact of (30) imply the bound

$$
n^{q} c_{n} \leqslant\left(\frac{n-k_{n}^{*}}{n}\right)^{-q} \sum_{k=0}^{k_{n}^{*}} c_{k} \leqslant\left(\frac{n-k_{n}^{*}}{n}\right)^{-q} W(q)=O\left(n^{q / 2}\right), \quad n \rightarrow \infty
$$

and so

$$
c_{n}=O\left(n^{-q / 2}\right), \quad n \rightarrow \infty .
$$

The first fact of (30) now produces $c_{k}^{(n-k-1)} \leqslant c_{k}=O\left(k^{-q / 2}\right)$, which implies that the third sum of (33) is of order

$$
O(1) \sum_{k=n^{*}+1}^{k_{n}^{*}}\left(\frac{n-k}{n} \sqrt{k}\right)^{-q} c_{k}=O(1)\left(\frac{\left(n-k_{n}^{*}\right) \sqrt{k_{n}^{*}}}{n}\right)^{-q} \sum_{k=n^{*}+1}^{k_{n}^{*}} c_{k} \rightarrow 0
$$

where the last step holds because $\lim _{n \rightarrow \infty} \frac{\left(n-k_{n}^{*}\right) \sqrt{k_{n}^{*}}}{n}=\sqrt{2}$ and because the last sum is the tail of a convergent series. Similarly, in the second sum, $\frac{n-k}{n} \geqslant \frac{n-n^{*}}{n}>1 / 2$, which implies that the second sum also vanishes as $n \rightarrow \infty$. As a result, the main contribution comes from the first sum, for which $\frac{n-k}{n} \uparrow 1$ termwise as $n \rightarrow \infty$, so that the limit of the first sum as $n \rightarrow \infty$ is $W(q)$ by dominated convergence.

Remark: Comparing the asymptotics of $c_{n}$ in the cases $0<q<1, q=1$ and $q>1$ it is clearly seen that $q=1$ is a point of phase transition.

\section{Appendix A The proof of Theorem 1}

The first step in the proof is to find the asymptotics of $\mathcal{F}(\delta):=f\left(e^{-\delta}\right)$, as $\delta \rightarrow 0^{+}$, because that will help us estimate $f_{n}\left(e^{-\delta}\right)$ for an appropriate choice of $\delta=\delta_{n}$. This is done in Lemma 1 below by using the Mellin transform method of Meinardus and Condition $(I)$ on the poles of $D(s)$. In the probabilistic approach initiated by Khintchine, the free parameter $\delta=\delta_{n}$ is chosen to be the solution of the equation

$$
\mathbb{E} U_{n}=n, n \geqslant 1 \text {. }
$$

We introduce the notations

$$
\begin{gathered}
\hat{h}_{l}=\rho_{l} h_{l}, \quad l=1, \ldots r, \\
\hat{h}_{0}=-A_{0},
\end{gathered}
$$

where the $h_{l}$ are defined in (45). In Proposition 2 below we use the fact that the equation (34) for $\delta_{n}$ can be written as

$$
\left(-\log \left(f_{n}\left(e^{-\delta}\right)\right)\right)_{\delta=\delta_{n}}^{\prime}=n, \quad n \geqslant 1
$$


to derive the facts (38), (39), and (40). In [7], by a careful analysis of the equation (37) the following expansion of $\delta_{n}, n \rightarrow \infty$ was derived:

$$
\delta_{n}=\left(\hat{h}_{r}\right)^{\frac{1}{\rho_{r}+1}} n^{-\frac{1}{\rho_{r}+1}}+\sum_{s=1}^{\left|\Upsilon_{r}\right|} \hat{K}_{s} n^{-\frac{1+\lambda_{s}}{\rho_{r}+1}}+o\left(n^{-1}\right),
$$

where $\hat{K}_{s}$ do not depend on $n$, and the powers $\lambda_{s}$ are as defined in (15). In [7] it was also shown that

$$
f_{n}\left(e^{-\delta_{n}}\right) \sim \mathcal{F}\left(\delta_{n}\right), \quad n \rightarrow \infty
$$

and, moreover, that

$$
\left(\log f_{n}\left(e^{-\delta}\right)\right)_{\delta=\delta_{n}}^{(k)}=(\log \mathcal{F}(\delta))_{\delta=\delta_{n}}^{(k)}+\epsilon_{k}(n),
$$

for $k=0,1,2,3$, where $\epsilon_{k}(n) \rightarrow 0, \quad n \rightarrow \infty, \quad k=0,1,2,3$.

We now analyze the three factors in the representation (19) when $\delta=\delta_{n}$ is given by (38).

(i) It follows from (38) that the first factor of (19) equals

$$
e^{n \delta_{n}}=\exp \left\{\left(\hat{h}_{r}\right)^{\frac{1}{\rho_{r}+1}} n^{\frac{\rho_{r}}{\rho_{r}+1}}+\sum_{s: \lambda_{s} \leqslant \rho_{r}} \tilde{K}_{s} n^{\frac{\rho_{r}-\lambda_{s}}{\rho_{r}+1}}+\epsilon_{n}\right\},
$$

where $\lambda_{s} \in \Upsilon_{r}$ and $\epsilon_{n} \rightarrow 0, n \rightarrow \infty$.

(ii) For $l=0,1, \ldots, r$

$$
\left(\delta_{n}\right)^{-\rho_{l}}=\left(\hat{h}_{r}\right)^{\frac{-\rho_{l}}{\rho_{r}+1}} n^{\frac{\rho_{l}}{\rho_{r}+1}}+\sum_{s: \lambda_{s} \leqslant \rho_{l}} K_{s, l} n^{\frac{\rho_{l}-\lambda_{s}}{\rho_{r}+1}}+\epsilon_{n}(l),
$$

where $\epsilon_{n}(l) \rightarrow 0, \quad n \rightarrow \infty, l=1,2, \ldots r$, and where the coefficients $K_{s, l}$ are obtained from the binomial expansion for $\left(\delta_{n}\right)^{-\rho_{l}}$, based on (38) and the definition (15) of the set $\Upsilon_{r}$. Consequently, substituting $\delta=\delta_{n}$ into the expression (44) for $\mathcal{F}(\delta)$ in Lemma 1 below gives

$$
\begin{gathered}
\log f_{n}\left(e^{-\delta_{n}}\right)=\sum_{l=0}^{r} \hat{h}_{l}\left(\hat{h}_{r}\right)^{\frac{-\rho_{l}}{\rho_{r}+1}} n^{\frac{\rho_{l}}{\rho_{r}+1}}+\sum_{l=0}^{r} \hat{h}_{l} \sum_{s: \lambda_{s} \leqslant \rho_{l}} K_{s, l} n^{\frac{\rho_{l}-\lambda_{s}}{\rho_{r}+1}}+ \\
\left(\frac{A_{0}}{\rho_{r}+1} \log n-\frac{A_{0}}{\rho_{r}+1} \log \hat{h}_{r}\right)+\epsilon_{n}, \epsilon_{n} \rightarrow 0, n \rightarrow \infty .
\end{gathered}
$$

(iii) The asymptotics of the third factor of (19) are given by Theorem 4, which is a local limit theorem, and which is proved using condition $(I I I)$. The proof uses ideas from [3]. As a result of Theorem 4, we have

$$
\mathbb{P}\left(U_{n}=n\right) \sim \frac{1}{\sqrt{2 \pi K_{2}}}\left(\hat{h}_{r}\right)^{\frac{2+\rho_{r}}{2\left(\rho_{r}+1\right)}} n^{-\frac{2+\rho_{r}}{2\left(\rho_{r}+1\right)}}, \quad n \rightarrow \infty
$$

for a constant $K_{2}=h_{r} \rho_{r}\left(\rho_{r}+1\right)$. 
Finally, to completely account for the influence of all $r+1$ poles $\rho_{0}, \rho_{1}, \ldots, \rho_{r}$, we present the sum of the expressions (41), (42) obtained in (i),(ii) for the first two factors in the representation (19) in the following form:

$$
\begin{aligned}
n \delta_{n}+\log f_{n}\left(e^{-\delta_{n}}\right)= & \sum_{l=0}^{r} P_{l} n^{\frac{\rho_{l}}{\rho_{r}+1}}+\sum_{l=0}^{r} h_{l} \sum_{s: \lambda_{s} \leqslant \rho_{l}} K_{s, l} n^{\frac{\rho_{l}-\lambda_{s}}{\rho_{r}+1}} \\
& +\left(\frac{A_{0}}{\rho_{r}+1} \log n-\frac{A_{0}}{\rho_{r}+1} \log \hat{h}_{r}\right)+\epsilon_{n},
\end{aligned}
$$

where $P_{l}$ denotes the resulting coefficient of $n^{\frac{\rho_{l}}{\rho_{r}+1}}$.

If $r=1$, then (37), (62) produce

$$
n=\hat{h}_{1} \delta_{n}^{-\rho_{r}-1}+\hat{h}_{0} \delta_{n}^{-1}+O\left(\delta_{n}^{C_{0}-1}\right)+\varepsilon(n),
$$

with $\varepsilon(n) \rightarrow 0, n \rightarrow \infty$, which is analogous to equation (54) of [5]. The previous equation can be inverted as in [5], giving

$$
\delta_{n}=\hat{h}_{1}^{\frac{1}{\rho_{1}+1}} n^{-\frac{1}{\rho_{1}+1}}+\frac{\hat{h}_{0}}{\rho_{1}+1} n^{-1}+O\left(n^{-1-\beta}\right),
$$

where

$$
\beta= \begin{cases}\frac{C_{0}}{\rho_{1}+1}, & \text { if } \rho_{1} \geqslant C_{0} \\ \frac{\rho_{1}}{\rho_{1}+1}, & \text { otherwise. }\end{cases}
$$

Substituting (43) into the previous asymptotic estimates of the three factors in (19), obtained in $(i)-(i i i)$, proves the asymptotic formula (17) for $c_{n}$.

We now present the results to which we refered in the summary above.

Lemma 1 (i) As $\delta \rightarrow 0^{+}$,

$$
\mathcal{F}(\delta)=\exp \left(\sum_{l=0}^{r} h_{l} \delta^{-\rho_{l}}-A_{0} \log \delta+M\left(\delta, C_{0}\right)\right),
$$

where $0<C_{0}<1, \rho_{0}=0$,

$$
\begin{aligned}
h_{l} & =A_{l} \Gamma\left(\rho_{l}\right), \quad l=1, \ldots, r, \\
h_{0} & =\Theta-\gamma A_{0}, \\
M\left(\delta ; C_{0}\right) & =\frac{1}{2 \pi i} \int_{-C_{0}-i \infty}^{-C_{0}+i \infty} \delta^{-s} \Gamma(s) D(s) d s=O\left(\delta^{C_{0}}\right), \delta \rightarrow 0,
\end{aligned}
$$

and where $\Theta$ is as in (18).

(ii) The asymptotic expressions for the derivatives

$$
(\log \mathcal{F}(\delta))^{(k)}
$$

are given by the formal differentiation of the logarithm of (44), with $\left(M\left(\delta ; C_{0}\right)\right)_{\delta}^{(k)}=O\left(\delta^{C_{0}-k}\right), k=1,2,3, \delta \rightarrow 0$. 
Proof We use the fact that $e^{-u}, u>0$, is the Mellin transform of the Gamma function:

$$
e^{-u}=\frac{1}{2 \pi i} \int_{v-i \infty}^{v+i \infty} u^{-s} \Gamma(s) d s, \quad u>0, \Re(s)=v>0 .
$$

Applying (46) with $v=\rho_{r}+\epsilon, \epsilon>0$ we have

$$
\begin{aligned}
\log \mathcal{F}(\delta) & =\sum_{k=1}^{\infty} b_{k} \log S\left(a_{k} e^{-\delta k}\right) \\
& =\sum_{k=1}^{\infty} b_{k} \sum_{j=1}^{\infty} \xi_{j} a_{k}^{j} e^{-\delta j k} \\
& =\sum_{k=1}^{\infty} \sum_{j=1}^{\infty} b_{k} \xi_{j} a_{k}^{j} \frac{1}{2 \pi i} \int_{\epsilon+\rho_{r}-i \infty}^{\epsilon+\rho_{r}+i \infty}(\delta j k)^{-s} \Gamma(s) d s \\
& =\frac{1}{2 \pi i} \int_{\epsilon+\rho_{r}-i \infty}^{\epsilon+\rho_{r}+\infty} \delta^{-s} \Gamma(s) \sum_{k=1}^{\infty} \sum_{j=1}^{\infty} b_{k} \xi_{j} a_{k}^{j}(j k)^{-s} d s \\
& =\frac{1}{2 \pi i} \int_{\epsilon+\rho_{r}-i \infty}^{\epsilon+\rho_{r}+i \infty} \delta^{-s} \Gamma(s) D(s) d s,
\end{aligned}
$$

where we have used (6),(7) and (5)-(8) at (47). Next, we apply the residue theorem for the integral (47), in the complex domain $-C_{0} \leqslant \Re(s) \leqslant \rho_{r}+\epsilon$, with $0<C_{0}<1, \epsilon>0$. By virtue of condition $(I)$, the integrand in (47) has in the above domain $r$ simple poles at $\rho_{l}>0, l=1, \ldots, r$. The corresponding residues at $s=\rho_{l}$ are equal to: $A_{l} \delta^{-\rho_{l}} \Gamma\left(\rho_{l}\right)$, $l=1, \ldots, r$.

By the Laurent expansions at $s=0$ of the Gamma function $\Gamma(s)=\frac{1}{s}-\gamma+\ldots$, and the function $D(s)=\frac{A_{0}}{s}+\Theta+\cdots$, the integrand $\delta^{-s} D(s) \Gamma(s)$ may also have a pole at $s=0$, which is a simple one with residue $\Theta$, if $A_{0}=0, \Theta \neq 0$, and is of a second order with residue $\Theta-\gamma A_{0}-A_{0} \log \delta$, if $A_{0} \neq 0$. In the case $A_{0}=\Theta=0$, the integrand $\delta^{-s} D(s) \Gamma(s)$ is analytic at $s=0$.

To apply the residue theorem, we bound the aforementioned domain by two horizontal contours $|\Im(s)|=t>0$. By condition $(I I)$, the integral of the integrand $\delta^{-s} \Gamma(s) D(s)$, over the horizontal contours $-C_{0} \leqslant \Re(s) \leqslant \epsilon+\rho_{r},|\Im(s)|=t>0$, tends to zero, as $t \rightarrow \infty$, for any fixed $\delta>0$. This gives the claimed formulae (44), where the remainder term $M\left(\delta ; C_{0}\right)$ is the integral taken over the vertical contour $-C_{0}+i t,-\infty<t<\infty$. This proves $(i)$.

In order to prove $(i i)$, one differentiates the logarithm of (44) with respect to $\delta$ and estimates the remaining integral in the same way as above.

We will need the following bound on $b_{k}$.

Proposition 1 Let the double series $D(s)$ defined by (8) converge absolutely in the halfplane $\mathcal{R}(s)>\rho$, for some $\rho>\rho_{r}$. Then the following bound holds

$$
b_{k} a_{k}^{l_{0}}=o\left(k^{\rho}\right), \quad k \rightarrow \infty,
$$

where $l_{0}$ is defined by (12). 
Proof We observe that $l_{0}$ defined by (12) satisfies

$$
l_{0}=\min \left\{j \geqslant 1: \xi_{j} \neq 0\right\} .
$$

The assumed absolute convergence of the double series in (8) implies the absolute convergence for $\rho>\rho_{r}$ of the iterated series

$$
\sum_{j=1}^{\infty} \frac{\xi_{j}}{j^{\rho}} \sum_{k=1}^{\infty} \frac{b_{k} a_{k}^{j}}{k^{\rho}}
$$

Consequently,

$$
\sum_{k=1}^{\infty} \frac{b_{k} a_{k}^{j}}{k^{\rho}}<\infty, \quad \text { for all } j \geqslant 1: \xi_{j} \neq 0
$$

Hence,

$$
\frac{b_{k} a_{k}^{j}}{k^{\rho}} \rightarrow 0, \quad k \rightarrow \infty, \quad \text { for all } j \geqslant 1: \xi_{j} \neq 0 .
$$

The latter implies (48).

Proposition 2 For sufficiently large $n$ there is a unique solution of (34) and that solution satisfies (38), (39), and (40).

Proof For each $n \geqslant 1$, the function $\left(-\log \left(f_{n}\left(e^{-\delta}\right)\right)\right)_{\delta}^{\prime}$ is decreasing for all $\delta>0$ because of (16). Moreover, setting

$$
\delta=\delta(n):=C n^{-\frac{1}{\rho_{r}+1}}, C>0
$$

we have

$$
\begin{aligned}
\left(-\log \left(f_{n}\left(e^{-\delta}\right)\right)\right)_{\delta(n)}^{\prime} & =(-\log \mathcal{F}(\delta))_{\delta(n)}^{\prime}-\sum_{k=n+1}^{\infty}\left(-b_{k} \log S\left(a_{k} e^{-k \delta}\right)\right)_{\delta(n)}^{\prime} \\
& =(-\log \mathcal{F}(\delta))_{\delta(n)}^{\prime}-O\left(\sum_{k=n+1}^{\infty} b_{k} \xi_{l_{0}} a_{k}^{l_{0}} e^{-\delta k l_{0}} k l_{0}\right) \\
& \sim C^{\frac{1}{\rho_{r}+1}} \rho_{r} h_{r} n, \quad C>0 .
\end{aligned}
$$

Here the step before the last follows because for $\delta$ defined by (50) we have $n \delta=C n^{\frac{\rho_{r}}{\rho_{r}+1}} \rightarrow$ $\infty, n \rightarrow \infty$, because of Lemma 1 (ii) and due to the fact that

$$
-\left(\log S\left(a_{k} e^{-k \delta}\right)\right)_{\delta(n)}^{\prime} \sim \xi_{l_{0}} a_{k}^{l_{0}} e^{-\delta(n) k l_{0}} k l_{0}, \quad n \rightarrow \infty
$$

uniformly for $k \geqslant n+1$, where $l_{0}$ satisfies (49). The last step in (51) results from Lemma 1 (ii) and (48). For $n$ sufficiently large, the right hand side of (51) is $>n$, if 
$C>\left(\rho_{r} h_{r}\right)^{-\left(\rho_{r}+1\right)}$ and $\leqslant n$ otherwise. This and (16) say that for a sufficiently large $n$, (37) has a unique solution $\delta_{n}$, which satisfies

$$
\delta_{n} \sim\left(\rho_{r} h_{r}\right)^{-\left(\rho_{r}+1\right)} n^{-\frac{1}{\rho_{r}+1}}, \quad n \rightarrow \infty,
$$

where $h_{r}$ is defined as in (44). We proceed to find an asymptotic expansion for $\delta_{n}$ by using a refinement of the scheme of Proposition 1 of [7]. We call any $\tilde{\delta}_{n}$, such that

$$
\left(-\log f_{n}\left(e^{-\delta}\right)\right)_{\delta=\tilde{\delta}_{n}}^{\prime}-n \rightarrow 0, \quad n \rightarrow \infty
$$

an asymptotic solution of (37). We will show that it is sufficient for (53) that $\tilde{\delta}_{n}$ obeys the condition

$$
(-\log \mathcal{F}(\delta))_{\delta=\tilde{\delta}_{n}}^{\prime}-n \rightarrow 0, n \rightarrow \infty
$$

By Lemma 1, we have

$$
(-\log \mathcal{F}(\delta))_{\delta}^{\prime} \sim h_{r} \rho_{r} \delta^{-\rho_{r}-1}, \delta \rightarrow 0
$$

so that (54) implies

$$
\tilde{\delta}_{n} \sim\left(h_{r} \rho_{r}\right)^{\frac{1}{\rho_{r}+1}} n^{-\frac{1}{\rho_{r}+1}}, n \rightarrow \infty .
$$

Next we have for all $n \geqslant 1$

$$
\log f_{n}\left(e^{-\tilde{\delta}_{n}}\right)=\log \mathcal{F}\left(\tilde{\delta}_{n}\right)-\sum_{k=n+1}^{\infty} b_{k} \log S\left(a_{k} e^{-k \tilde{\delta}_{n}}\right) .
$$

Applying the same argument as in (51), we derive the bound

$$
\sum_{k=n+1}^{\infty}\left(-b_{k} \log S\left(a_{k} e^{-k \delta}\right)\right)_{\delta=\tilde{\delta}_{n}}^{\prime}=o(1), \quad n \rightarrow \infty .
$$

Now, (56) and (57) show that (54) implies (53). We will now demonstrate that the error of approximating the exact solution $\delta_{n}$ by the asymptotic solution $\tilde{\delta}_{n}$ is of order $o\left(n^{-1}\right)$. By the definitions of $\delta_{n}, \tilde{\delta}_{n}$ we have

$$
\left(-\log f_{n}\left(e^{-\delta}\right)\right)_{\delta=\delta_{n}}^{\prime}-\left(-\log f_{n}\left(e^{-\delta}\right)\right)_{\delta=\tilde{\delta}_{n}}^{\prime}=\epsilon_{n}, \quad \epsilon_{n} \rightarrow 0, \quad n \rightarrow \infty .
$$

Next, applying the Mean Value Theorem, we obtain

$$
\left|\left(-\log f_{n}\left(e^{-\delta_{n}}\right)\right)^{\prime}-\left(-\log f_{n}\left(e^{-\tilde{\delta}_{n}}\right)\right)^{\prime}\right|=\left|\left(\delta_{n}-\tilde{\delta}_{n}\right)\left(\log f_{n}\left(e^{-u_{n}}\right)\right)^{\prime \prime}\right|,
$$

where

$$
u_{n} \in\left[\min \left(\delta_{n}, \tilde{\delta}_{n}\right), \max \left(\delta_{n}, \tilde{\delta}_{n}\right)\right] .
$$

By (58), the left hand side of (59) tends to 0 , as $n \rightarrow \infty$, while, by virtue of (52),(55),

$$
\left(\log f_{n}\left(e^{-\delta}\right)\right)_{u_{n}}^{\prime \prime} \sim \rho_{r}\left(\rho_{r}+1\right) h_{r}\left(\delta_{n}\right)^{-\rho_{r}-2}=O\left(n^{\frac{\rho_{r}+2}{\rho_{r}+1}}\right), \quad n \rightarrow \infty .
$$


Combining (59) with (60), gives the desired estimate

$$
\left|\delta_{n}-\tilde{\delta}_{n}\right|=o\left(n^{-1}\right), \quad n \rightarrow \infty .
$$

An obvious modification of the argument in (51) allows also to conclude that

$$
\sum_{k=n+1}^{\infty} b_{k} \log S\left(a_{k} e^{-k \delta_{n}}\right) \rightarrow 0, \quad n \rightarrow \infty .
$$

As a result, (39) is valid.

By $(i i)$ of Lemma 1 we have

$$
(-\log \mathcal{F}(\delta))_{\delta}^{\prime}=\sum_{l=0}^{r} \hat{h}_{l} \delta^{-\rho_{l}-1}+\left(M\left(\delta ; C_{0}\right)\right)_{\delta}^{\prime} .
$$

This is exactly the starting point of the analysis of $\tilde{\delta}_{n}$ in Proposition 1 of [7]. We may therefore apply Proposition 1 of [7], which provides detailed asymptotics for $\delta_{n}$, and (61), to conclude that (38) holds.

Finally, by an argument similar to the one for the proof of (57) we see that (40) holds, as well.

The following estimate is central to our proof of Theorem 4.

Proposition 3 Recall that $\phi_{n}(\alpha)$ is defined by (21) and that $l_{0}$ is defined by (12). Then we have for all $\alpha \in \mathcal{R}$,

$$
\begin{aligned}
\log \left|\phi_{n}(\alpha)\right| & =\log \left|\phi_{n}\left(\alpha ; \delta_{n}\right)\right|=-2 \sum_{k=1}^{\infty} \Lambda_{k} e^{-k \delta_{n}} \sin ^{2}(\pi k \alpha)+\epsilon_{n} \\
& \leqslant-\frac{2 d_{l_{0}}}{S^{2}\left(e^{-1 / 8 l_{0}}\right)} \sum_{k=\left(8 l_{0} \delta_{n}\right)^{-1}}^{n} b_{k} a_{k}^{l_{0}} e^{-\delta_{n} l_{0} k} \sin ^{2}\left(\pi \alpha l_{0} k\right),
\end{aligned}
$$

where $\epsilon_{n} \rightarrow 0$ as $n \rightarrow \infty$.

Proof We write $\log \left|\phi_{n}(\alpha)\right|, \alpha \in \mathcal{R}$, as

$$
\begin{aligned}
\log \left|\phi_{n}(\alpha)\right|= & \frac{1}{2} \sum_{k=1}^{n} b_{k}\left\{\log S\left(a_{k} e^{2 \pi i k \alpha-k \delta_{n}}\right)+\log S\left(a_{k} e^{-2 \pi i k \alpha-k \delta_{n}}\right)-2 \log S\left(a_{k} e^{-k \delta_{n}}\right)\right\} \\
= & \frac{1}{2} \sum_{k=1}^{\infty} b_{k}\left\{\log S\left(a_{k} e^{2 \pi i k \alpha-k \delta_{n}}\right)+\log S\left(a_{k} e^{-2 \pi i k \alpha-k \delta_{n}}\right)-2 \log S\left(a_{k} e^{-k \delta_{n}}\right)\right\} \\
& +O\left(\sum_{k=n+1}^{\infty} b_{k} \xi_{l_{0}} a_{k}^{l_{0}} e^{-k \delta_{n} l_{0}}\right)
\end{aligned}
$$




$$
\begin{aligned}
= & \frac{1}{2} \sum_{k=1}^{\infty} b_{k}\left\{\log S\left(a_{k} e^{2 \pi i k \alpha-k \delta_{n}}\right)+\log S\left(a_{k} e^{-2 \pi i k \alpha-k \delta_{n}}\right)-2 \log S\left(a_{k} e^{-k \delta_{n}}\right)\right\} \\
& +o\left(\sum_{k=n+1}^{\infty} k^{\rho} e^{-k \delta_{n} l_{0}}\right) \\
= & \frac{1}{2} \sum_{k=1}^{\infty} b_{k} \sum_{j=1}^{\infty} \xi_{j} a_{k}^{j} e^{-j k \delta_{n}}\left(e^{2 \pi i j k \alpha}+e^{-2 \pi i j k \alpha}-2\right)+\epsilon_{n} \\
= & -2 \sum_{k=1}^{\infty} \sum_{j=1}^{\infty} b_{k} \xi_{j} a_{k}^{j} e^{-j k \delta_{n}} \sin ^{2}(\pi j k \alpha)+\epsilon_{n} \\
= & -2 \sum_{\ell=1}^{\infty} \Lambda_{\ell} e^{-\ell \delta_{n}} \sin ^{2}(\pi \ell \alpha)+\epsilon_{n}
\end{aligned}
$$

where $\epsilon_{n} \rightarrow 0, n \rightarrow \infty,(65)$ and (66) use (48), (67) uses (52) which implies $k^{\rho} e^{-k \delta_{n} l_{0}} \rightarrow$ $0, n \rightarrow \infty, k \geqslant n+1$ and any $\rho>0$. Finally, (68) follows from (6).

As for the inequality (64), defining $\tau$ to be $\tau=\delta_{n}-2 \pi i \alpha, \alpha \in \mathbb{R}$, we have

$$
\begin{aligned}
\log \left|\phi_{n}(\alpha)\right| & =\Re\left(\log f_{n}\left(e^{-\tau}\right)-\log f_{n}\left(e^{-\delta_{n}}\right)\right) \\
& =\frac{1}{2} \sum_{k=1}^{n} b_{k} \log \frac{\left|S\left(a_{k} e^{-k \tau}\right)\right|^{2}}{S^{2}\left(a_{k} e^{-k \delta_{n}}\right)} \\
& =\frac{1}{2} \sum_{k=1}^{n} b_{k} \log \left(1-\frac{S^{2}\left(a_{k} e^{-k \delta_{n}}\right)-\left|S\left(a_{k} e^{-k \tau}\right)\right|^{2}}{S^{2}\left(a_{k} e^{-k \delta_{n}}\right)}\right) \\
& \leqslant-\frac{1}{2} \sum_{k=1}^{n} b_{k} \frac{S^{2}\left(a_{k} e^{-k \delta_{n}}\right)-\left|S\left(a_{k} e^{-k \tau}\right)\right|^{2}}{S^{2}\left(a_{k} e^{-k \delta_{n}}\right)},
\end{aligned}
$$

where the last inequality is because $S^{2}\left(a_{k} e^{-k \delta_{n}}\right)-\left|S\left(a_{k} e^{-k \tau}\right)\right|^{2} \geqslant 0$, for all $\alpha \in \mathcal{R}$ and because $\log (1-x) \leqslant-x, 0<x<1$. Recalling (4) and (12), we obtain for all $\alpha \in \mathcal{R}$,

$$
\begin{aligned}
S^{2}\left(a_{k} e^{-k \delta_{n}}\right)-\left|S\left(a_{k} e^{-k \tau}\right)\right|^{2} & =4 \sum_{0 \leqslant l, m<\infty} d_{l} d_{m} a_{k}^{l+m} e^{-(l+m) k \delta_{n}} \sin ^{2}((l-m) \pi \alpha k) \\
& \geqslant 4 d_{l_{0}} a_{k}^{l_{0}} e^{-\delta_{n} l_{0} k} \sin ^{2}\left(\pi \alpha l_{0} k\right), k=1,2, \ldots
\end{aligned}
$$

which allows us to continue (69), arriving at the desired bound:

$$
\begin{aligned}
\log \left|\phi_{n}(\alpha)\right| & \leqslant-2 d_{l_{0}} \sum_{k=1}^{n} b_{k} a_{k}^{l_{0}} \frac{e^{-\delta_{n} l_{0} k} \sin ^{2}\left(\pi \alpha l_{0} k\right)}{S^{2}\left(a_{k} e^{-k \delta_{n}}\right)} \\
& \leqslant-2 d_{l_{0}} \sum_{k=\left(8 l_{0} \delta_{n}\right)^{-1}}^{n} b_{k} a_{k}^{l_{0}} \frac{e^{-\delta_{n} l_{0} k} \sin ^{2}\left(\pi \alpha l_{0} k\right)}{S^{2}\left(a_{k} e^{-k \delta_{n}}\right)} \\
& \leqslant-\frac{2 d_{l_{0}}}{S^{2}\left(e^{-1 / 8 l_{0}}\right)} \sum_{k=\left(8 l_{0} \delta_{n}\right)^{-1}}^{n} b_{k} a_{k}^{l_{0}} e^{-\delta_{n} l_{0} k} \sin ^{2}\left(\pi \alpha l_{0} k\right),
\end{aligned}
$$


where the last step is because $d_{l} \geqslant 0, l=1,2, \ldots$, because $0<a_{k} \leqslant 1$ and because $1 \leqslant S(z)<\infty$ is monotonically increasing in $0 \leqslant z<1$.

Theorem 4 (Local Limit Theorem) Let the random variable $U_{n}$ be defined as in (20), (21). Then

$$
\begin{aligned}
\mathbb{P}\left(U_{n}=n\right) & \sim \frac{1}{\sqrt{2 \pi \operatorname{Var}\left(\mathrm{U}_{\mathrm{n}}\right)}} \\
& \sim \frac{1}{\sqrt{2 \pi K_{2}}}\left(\delta_{n}\right)^{1+\rho_{r} / 2} \\
& \sim \frac{1}{\sqrt{2 \pi K_{2}}}\left(\hat{h}_{r}\right)^{\frac{2+\rho_{r}}{2\left(\rho_{r}+1\right)}} n^{-\frac{2+\rho_{r}}{2\left(\rho_{r}+1\right)}}, \quad n \rightarrow \infty
\end{aligned}
$$

for a constant $K_{2}=h_{r} \rho_{r}\left(\rho_{r}+1\right)$.

Proof We take $\delta=\delta_{n}$ in (21) and define

$$
\alpha_{0}=\alpha_{0}(n)=\left(\delta_{n}\right)^{\frac{\rho_{r}+2}{2}} \log n .
$$

We write

$$
\mathbb{P}\left(U_{n}=n\right)=\int_{-1 / 2}^{1 / 2} \phi_{n}(\alpha) e^{-2 \pi i n \alpha} d \alpha=I_{1}+I_{2},
$$

where

$$
I_{1}=\int_{-\alpha_{0}}^{\alpha_{0}} \phi_{n}(\alpha) e^{-2 \pi i n \alpha} d \alpha
$$

and

$$
I_{2}=\int_{-1 / 2}^{-\alpha_{0}} \phi_{n}(\alpha) e^{-2 \pi i n \alpha} d \alpha+\int_{\alpha_{0}}^{1 / 2} \phi_{n}(\alpha) e^{-2 \pi i n \alpha} d \alpha .
$$

The proof has two parts corresponding to evaluation of the integrals $I_{1}$ and $I_{2}$, as $n \rightarrow \infty$.

Part 1: Integral $I_{1}$. Defining $B_{n}$ and $T_{n}$ by

$$
B_{n}^{2}=\left(\log f_{n}\left(e^{-\delta}\right)\right)_{\delta=\delta_{n}}^{\prime \prime} \text { and } T_{n}=-\left(\log f_{n}\left(e^{-\delta}\right)\right)_{\delta=\delta_{n}}^{\prime \prime \prime}
$$

for $n$ fixed we have the expansion in $\alpha$

$$
\begin{aligned}
\phi_{n}(\alpha) e^{-2 \pi i n \alpha} & =\exp \left(2 \pi i \alpha\left(\mathbb{E} U_{n}-n\right)-2 \pi^{2} \alpha^{2} B_{n}^{2}+O\left(\alpha^{3}\right) T_{n}\right) \\
& =\exp \left(-2 \pi^{2} \alpha^{2} B_{n}^{2}+O\left(\alpha^{3}\right) T_{n}\right), \quad \alpha \rightarrow 0,
\end{aligned}
$$

where the second equation is due to (34). By virtue of (44) and (40) we derive from (71) that the main terms in the asymptotics for $B_{n}^{2}$ and $T_{n}$ depend on the rightmost pole $\rho_{r}$ only:

$$
B_{n}^{2} \sim K_{2}\left(\delta_{n}\right)^{-\rho_{r}-2}
$$




$$
T_{n} \sim K_{3}\left(\delta_{n}\right)^{-\rho_{r}-3}, \quad n \rightarrow \infty
$$

where $K_{2}=h_{r} \rho_{r}\left(\rho_{r}+1\right)$ and $K_{3}=h_{r} \rho_{r}\left(\rho_{r}+1\right)\left(\rho_{r}+2\right)$ are obtained from (71) and Lemma 1. Therefore, by virtue of (70),

$$
B_{n}^{2} \alpha_{0}^{2} \rightarrow \infty, T_{n} \alpha_{0}^{3} \rightarrow 0, n \rightarrow \infty .
$$

Consequently, in the same way as in the proof of the local limit theorem in [5],

$$
I_{1} \sim \frac{1}{\sqrt{2 \pi B_{n}^{2}}}, n \rightarrow \infty
$$

and it is left to show that

$$
I_{2}=o\left(I_{1}\right), n \rightarrow \infty .
$$

Part 2: Integral $I_{2}$. We rewrite the upper bound in (64) in Proposition 3 as

$$
\log \left|\phi_{n}(\alpha)\right| \leqslant-C V_{n}(\alpha), \quad \alpha \in \mathbb{R}
$$

where $C>0$ does not depend on $n$ and

$$
V_{n}(\alpha):=\sum_{k=\left(8 l_{0} \delta_{n}\right)^{-1}}^{n} b_{k} a_{k}^{l_{0}} e^{-\delta_{n} l_{0} k} \sin ^{2}\left(\pi \alpha l_{0} k\right) .
$$

It is enough to consider $\alpha \geqslant 0$, as the case $\alpha<0$ is exactly the same.

We split the interval of integration $\left[\alpha_{0}, 1 / 2\right]$ into subintervals:

$$
\left[\alpha_{0},(2 \pi)^{-1} \delta_{n}\right] \cup\left[(2 \pi)^{-1} \delta_{n}, 1 / 2\right] \text { if } l_{0}=1
$$

and

$$
\left[\alpha_{0},\left(2 \pi l_{0}\right)^{-1} \delta_{n}\right] \cup\left[\left(2 \pi l_{0}\right)^{-1} \delta_{n},\left(2 l_{0}\right)^{-1}\right] \cup\left[\left(2 l_{0}\right)^{-1}, 1 / 2\right] \text { if } l_{0}>1 .
$$

Our goal is to bound, as $n \rightarrow \infty$, the function $V_{n}(\alpha)$ from below in each of the subintervals. Firstly, we show that on the first two subintervals for $l_{0} \geqslant 1$, the desired bound is implied by the assumption (11) in condition $(I I I)$.

In the first subinterval $\left[\alpha_{0},\left(2 l_{0}\right)^{-1} \delta_{n}\right], l_{0} \geqslant 1$ we will use the inequality

$$
\sin ^{2}(\pi x) \geqslant 4\|x\|^{2}, x \in \mathbb{R},
$$

where $\|x\|$ denotes the distance from $x$ to the nearest integer, i.e.

$$
\|x\|= \begin{cases}\{x\} & \text { if }\{x\} \leqslant 1 / 2 \\ 1-\{x\} & \text { if }\{x\}>1 / 2\end{cases}
$$

(see [3] for the proof of (75)). By (11) and (75), we then have

$$
V_{n}(\alpha) \geqslant 4 e^{-1 / 2} \sum_{k=\left(4 l_{0} \delta_{n}\right)^{-1}}^{\left(2 l_{0} \delta_{n}\right)^{-1}} C_{2} k^{\rho_{r}-1}\left\|\alpha l_{0} k\right\|^{2}, \alpha \in \mathbb{R}, l_{0} \geqslant 1 .
$$


In the first subinterval,

$$
\left\|\alpha l_{0} k\right\|=\alpha l_{0} k, 1 \leqslant k \leqslant\left(2 l_{0} \delta_{n}\right)^{-1}, l_{0} \geqslant 1,
$$

so that (76) and (70) produce

$$
\begin{aligned}
V_{n}(\alpha) & \geqslant 4 C_{2} e^{-1 / 2} l_{0}^{2} \alpha_{0}^{2} \sum_{k=\left(4 l_{0} \delta_{n}\right)^{-1}}^{\left(2 l_{0} \delta_{n}\right)^{-1}} k^{\rho_{r}+1} \\
& \sim 4 C_{2} e^{-1 / 2}\left(\rho_{r}+2\right)^{-1} l_{0}^{2} \alpha_{0}^{2}\left(\left(2 l_{0} \delta_{n}\right)^{-\rho_{r}-2}-\left(4 l_{0} \delta_{n}\right)^{-\rho_{r}-2}\right), \quad n \rightarrow \infty .
\end{aligned}
$$

By (70) and (64) this gives the desired bound in the first subinterval:

$$
\log \left|\phi_{n}(\alpha)\right| \leqslant-C \log ^{2} n, \quad C>0, \quad n \rightarrow \infty .
$$

For the second subinterval we will apply the argument in the proof of Lemma 1 in [5]. Given $\alpha \in \mathbb{R} \backslash\{0\}$, define the function $P\left(\alpha, \delta_{n}\right)$ by

$$
P\left(\alpha, \delta_{n}\right)=\left[\frac{1+|\alpha| \delta_{n}^{-1}}{2|\alpha|}\right] \geqslant 1,
$$

where $[x]$ denotes the integer part of $x$ and the inequality holds for $n$ large enough. The lower bound for the zeta sum (see (17) in [5]), supplies the bound

$$
\sum_{k=\left(8 \delta_{n}\right)^{-1}}^{P\left(\alpha, \delta_{n}\right)} \sin ^{2}(\pi k \alpha) \geqslant \frac{\delta_{n}^{-1}}{8},
$$

provided

$$
\frac{\delta_{n}}{2 \pi} \leqslant \alpha \leqslant 1 / 2
$$

Observing, that by definition (78), $n>P\left(l_{0} \alpha, \delta_{n}\right)>\left(2 \delta_{n}\right)^{-1}>\left(8 \delta_{n}\right)^{-1}$ we rewrite (79) as

$$
\sum_{k=\left(8 l_{0} \delta_{n}\right)^{-1}}^{P\left(l_{0} \alpha, \delta_{n}\right)} \sin ^{2}\left(\pi k l_{0} \alpha\right) \geqslant \frac{\delta_{n}^{-1}}{8}, l_{0} \geqslant 1
$$

for

$$
\left(2 \pi l_{0}\right)^{-1} \delta_{n} \leqslant \alpha \leqslant\left(2 l_{0}\right)^{-1}, \quad l_{0} \geqslant 1 .
$$

We now write $P$ for $P\left(l_{0} \alpha, \delta_{n}\right)$. For $\alpha \in \mathbb{R}$ and $l_{0} \geqslant 1$, we have

$$
\begin{aligned}
\sum_{k=\left(8 l_{0} \delta_{n}\right)^{-1}}^{n} b_{k} a_{k}^{l_{0}} e^{-\delta_{n} l_{0} k} \sin ^{2}\left(\pi \alpha l_{0} k\right) & \geqslant \sum_{k=\left(8 l_{0} \delta_{n}\right)^{-1}}^{P} C_{2} k^{\rho_{r}-1} e^{-\delta_{n} l_{0} k} \sin ^{2}\left(\pi \alpha l_{0} k\right) \\
& \geqslant C_{2} e^{-P l_{0} \delta_{n}} \sum_{k=\left(8 l_{0} \delta_{n}\right)^{-1}}^{P} k^{\rho_{r}-1} \sin ^{2}\left(\pi \alpha l_{0} k\right) \\
& :=Q(\alpha) .
\end{aligned}
$$


In order to get the needed lower bound on $Q(\alpha)$, we take into account that for all $\alpha$ obeying (82), $\frac{1}{2}<P \delta_{n}<\frac{1}{2}(1+2 \pi):=d$. Applying (81), we distinguish between the following two cases: $(i) 0<\rho_{r}<1$ and $($ ii $) \rho_{r} \geqslant 1$.

For $\alpha$ in (82), we have in case $(i)$,

$$
Q(\alpha) \geqslant C_{2} e^{-P l_{0} \delta_{n}} P^{\rho_{r}-1}\left(8 \delta_{n}\right)^{-1} \geqslant \frac{C_{2}}{8} e^{-d l_{0}}\left(P \delta_{n}\right)^{\rho_{r}-1} \delta_{n}^{-\rho_{r}} \geqslant \frac{C_{2}}{8} d^{\rho_{r}-1} e^{-d l_{0}} \delta_{n}^{-\rho_{r}}:=C_{3} \delta_{n}^{-\rho_{r}},
$$

and in case $(i i)$,

$$
Q(\alpha) \geqslant C_{2} e^{-P l_{0} \delta_{n}}\left(8 \delta_{n}\right)^{-\rho_{r}+1}\left(8 l_{0} \delta_{n}\right)^{-1} \geqslant C_{2} e^{-d l_{0}} 8^{-\rho_{r}} \delta_{n}^{-\rho_{r}} l_{0}^{-1}:=C_{4} \delta_{n}^{-\rho_{r}} .
$$

Finally, combining this with (64) gives the desired upper bound on $\log \left|\phi_{n}(\alpha)\right|$ for all $\alpha$ in (82) and $n$ sufficiently large:

$$
\log \left|\phi_{n}(\alpha)\right| \leqslant-C \delta_{n}^{-\rho_{r}}, C>0 .
$$

Remark: If $l_{0}>1$, then $\sin ^{2}\left(\pi \alpha l_{0} k\right)=0, k \geqslant 1$ when $\alpha=l_{0}^{-1} \leqslant 1 / 2$, so that in the third subinterval $\left[\left(2 l_{0}\right)^{-1}, 1 / 2\right]$ the above bounds are not applicable.

In the third subinterval $\left[\left(2 l_{0}\right)^{-1}, 1 / 2\right], l_{0}>1$ we apply (13) in condition $(I I I)$. By (63) and (13) we have for $n$ large enough,

$$
\left|\phi_{n}(\alpha)\right| \leqslant \delta_{n}^{1+\frac{\rho_{r}}{2}+\epsilon}, \epsilon>0 .
$$

Comparing the bounds (77), (83), (84) with the asymptotics (73), (72) proves (74).

\section{Appendix B A representation of $W(q)$}

In this appendix we derive representations of the function $W(q)$ in the case of rational $q>1$. The infinite product

$$
F(z):=\prod_{k=1}^{\infty}\left(1+\frac{z}{k^{q}}\right), \quad z \in \mathbb{C}, \quad q>1,
$$

is a Weierstrass representation of an entire function $F$ with zeroes at $\left\{-k^{q}, k=1,2, \ldots\right\}$. This follows from Theorem 5.12 in [1], since $\sum_{k=1}^{\infty} k^{-q}<\infty, q>1$. Note that $W(q)=$ $f(1)=F(1), q>1$. We now show that in the case when $q>1$ is a rational number, a modification of the argument in [17], p.238 allows us to decompose the value $F(1)$ in (85) into a finite product of values of a canonic entire function of finite rank. (For the definition of a rank of entire function see Chapter $X I$ in [1]). Let $q=\frac{m_{1}}{m_{2}}$, where $m_{1}>m_{2} \geqslant 1$ are co-prime integers. We write

$$
1+k^{-\frac{m_{1}}{m_{2}}}=\prod_{l=1}^{m_{1}} \frac{k^{\frac{1}{m_{2}}}-\alpha_{l}\left(m_{1}\right)}{k^{\frac{1}{m_{2}}}}=\prod_{l=1}^{m_{1}}\left(1-\frac{\alpha_{l}\left(m_{1}\right)}{k^{\frac{1}{m_{2}}}}\right),
$$


where

$$
\alpha_{l}\left(m_{1}\right)=\exp \left(\frac{\pi(2 l-1)}{m_{1}} i\right), \quad l=1, \ldots, m_{1}
$$

are all $m_{1}$-th roots of -1 , such that $0<\arg \left(\alpha_{l}\left(m_{1}\right)\right)<2 \pi, l=1, \ldots, m_{1}$.

Consequently,

$$
W(q)=\prod_{k=1}^{\infty} \prod_{l=1}^{m_{1}}\left(1-\frac{\alpha_{l}\left(m_{1}\right)}{k^{\frac{1}{m_{2}}}}\right), \quad q=\frac{m_{1}}{m_{2}} .
$$

Next, introduce the function

$$
\tilde{f}(z):=\prod_{k=1}^{\infty}\left(1+\frac{z}{k^{\frac{1}{m_{2}}}}\right) \exp \left(\sum_{p=1}^{m_{2}} \frac{(-z)^{p}}{k^{\frac{p}{m_{2}}} p}\right), \quad z \in \mathbb{C},
$$

which is a canonical form of an entire function of finite rank $m_{2}$ with zeroes

$$
\left\{-k^{\frac{1}{m_{2}}}, \quad k=1,2, \ldots\right\} .
$$

Observing that $\sum_{l=1}^{m_{1}}\left(\alpha_{l}\left(m_{1}\right)\right)^{p}=0, p=1, \ldots, m_{2}$, by the definition of $\alpha_{l}\left(m_{1}\right), l=$ $1, \ldots, m_{1}$, and rewriting (87) as

$$
\tilde{f}(z):=\prod_{k=1}^{\infty}\left(1+\frac{z}{k^{\frac{1}{m_{2}}}}\right) \prod_{p=1}^{m_{2}} \exp \left(\frac{(-z)^{p}}{k^{\frac{p}{m_{2}}} p}\right)
$$

we derive from (86):

$$
W(q)=\prod_{l=1}^{m_{1}} \tilde{f}\left(-\alpha_{l}\left(m_{1}\right)\right)
$$

for rational $q>1$.

For $m_{2}>1$, we will consider now the function

$$
\tilde{\Gamma}(z):=e^{Q(z)} \frac{1}{z \tilde{f}(z)},
$$

where $Q(z)$ is a polynomial in $z$ that will be defined below. The preceding discussion yields that $\tilde{\Gamma}$ is a meromorphic function in $\mathbb{C}$ with simple poles at $\left(-k^{\frac{1}{m_{2}}}\right), k=0,1, \ldots$ Now our purpose will be to obtain for the function $\tilde{\Gamma}$ an analog of Gauss formula for the gamma function. We recall the definition of generalized Euler constants:

$$
\begin{aligned}
\gamma_{\alpha} & =\lim _{n \rightarrow \infty}\left(\sum_{k=1}^{n} k^{-\alpha}-\int_{1}^{n} x^{-\alpha} d x\right) \\
& =\lim _{n \rightarrow \infty} \begin{cases}\sum_{k=1}^{n} \frac{1}{k}-\log n, & \text { if } \alpha=1 ; \\
\sum_{k=1}^{n} \frac{1}{k^{\alpha}}-\frac{n^{1-\alpha}-1}{1-\alpha}, & \text { if } \quad 0<\alpha<1 .\end{cases}
\end{aligned}
$$


(Note that $\gamma_{1}=\gamma$ is the standard Euler constant). This allows us to write the function $\frac{1}{z \tilde{f}(z)}, z \in \mathbb{C}$ in the following form:

$$
\begin{aligned}
\frac{1}{z \tilde{f}(z)}= & \lim _{n \rightarrow \infty} \frac{1}{z} \prod_{k=1}^{n}\left(\frac{k^{\frac{1}{m_{2}}}}{z+k^{\frac{1}{m_{2}}}}\right) \exp \left(-\sum_{p=1}^{m_{2}} \frac{(-z)^{p}}{k^{\frac{p}{m_{2}}} p}\right) \\
= & \exp \left(-\sum_{p=1}^{m_{2}}(-1)^{p^{\frac{z^{p}}{p}}} \gamma_{\left(p / m_{2}\right)}\right) \\
& \times \lim _{n \rightarrow \infty} \frac{(n !)^{\frac{1}{m_{2}}}}{\prod_{k=0}^{n}\left(z+k^{\frac{1}{m_{2}}}\right)} n^{\frac{-(-z)^{m_{2}}}{m_{2}}} \exp \left(-m_{2} \sum_{p=1}^{m_{2}-1} \frac{(-1)^{p} z^{p}\left(n^{\frac{m_{2}-p}{m_{2}}}-1\right)}{p\left(m_{2}-p\right)}\right) .
\end{aligned}
$$

Setting now in (89) $Q(z)=\sum_{p=1}^{m_{2}}(-1)^{p} \frac{z^{p}}{p} \gamma_{\left(p / m_{2}\right)}$, we arrive at the desired representation of the function

$$
\tilde{\Gamma}(z)=\lim _{n \rightarrow \infty} \frac{(n !)^{\frac{1}{m_{2}}}}{\prod_{k=0}^{n}\left(z+k^{\frac{1}{m_{2}}}\right)} n^{\frac{-(-z)^{m_{2}}}{m_{2}}} \exp \left(-m_{2} \sum_{p=1}^{m_{2}-1} \frac{(-1)^{p} z^{p}\left(n^{\frac{m_{2}-p}{m_{2}}}-1\right)}{p\left(m_{2}-p\right)}\right) .
$$

Under $m_{2}=1$, (90) becomes the Gauss formula for the gamma function.

In the case $q>1$ is an integer, (88) conforms to the explicit expression for $W(q)$ in [17], p.238-239. In fact, after substituting in (87) $m_{2}=1$ and $p=1$ we have

$$
\tilde{f}(z)=\prod_{k=1}^{\infty}\left(1+\frac{z}{k}\right) e^{-\frac{z}{k}}, \quad z \in \mathbb{C}
$$

and by the Weierstrass factorization theorem for the Gamma function,

$$
\tilde{f}(z)=\frac{e^{-\gamma z}}{\Gamma(1+z)}, \quad z \in \mathbb{C} \backslash\{-1,-2, \ldots\} .
$$

where $\gamma$ is Euler's constant. Thus, when $q>1$ is an integer,

$$
W(q)=\prod_{l=1}^{q}\left(\Gamma\left(1-\alpha_{l}(q)\right)\right)^{-1}
$$

Taking into account that the numbers $\alpha_{l}(q), l=1, \ldots, q$ are pairwise conjugate and that $\Gamma(\bar{z})=\overline{\Gamma(z)}, z \in \mathbb{C}$, the last expression can be written as follows:

$$
W(q)=\prod_{l=1}^{[q / 2]}\left(\left|\Gamma\left(1-\alpha_{l}(q)\right)\right|^{2}\right)^{-1}, \quad q>1
$$

\section{Acknowledgements}

Our paper has benefited by the remarks of an anonymous referee who, among other things, pointed an error in the original proof of the case $q>1$ in Theorem 3 . 


\section{References}

[1] J. B. Conway. Functions of one complex variable. Springer-Verlag, 1978.

[2] P. Flajolet, E. Fusy, X. Gourdon, D. Panario, and N. Pouyanne. A hybrid of Darboux's method and singularity analysis in combinatorial asymptotics. Electronic $J$. Combinatorics, 13 \#R103:1-35, 2006.

[3] G. Freiman and B. Granovsky. Asymptotic formula for a partition function of reversible coagulation-fragmentation processes. Israel J. Math., 130:259-279, 2002.

[4] G. Gentile. Osservazione sopra le statistiche intermedie. Nuovo Cimento, 17:493, 1941.

[5] B. Granovsky, D. Stark, and M. Erlihson. Meinardus' theorem on weighted partitions: Extensions and a probabilistic proof. Adv. Appl. Math., 41:307-328, 2008.

[6] B. Granovsky and D. Stark. Asymptotic enumeration and logical limit laws for expansive multisets. J. London Math. Soc. (2), 73:252-272, 2006.

[7] B. Granovsky and D. Stark. A Meinardus theorem with multiple singularities. Comm. Math. Phys., 314:329-350, 2012.

[8] D. H. Greene and D. E. Knuth. Mathematics for the Analysis of Algorithms. Third Edition, Birkhauser, 1990.

[9] G. H. Hardy and S. Ramanujan, S. Asymptotic formulae in combinatory analysis. Proc. London Math. Soc., 17:75-115, 1918.

[10] A. I. Khintchine. Mathematical foundations of quantum statistics. Graylock Press, Albany, N.Y., 1960.

[11] G. Meinardus. Asymptotische Aussagen über Partitionen. Math. Zeitschrift, 59:388398, 1954.

[12] A. M. Odlyzko. Asymptotic enumeration methods. In Handbook of Combinatorics, vol. II. R. Graham, M. Grötschel, and L. Lovász, Eds., pages 1063-1299, Elsevier 1995.

[13] C. S. Srivatsan, M. V. Murthy and R. K. Bhaduri. Gentile statistics and restricted partitions, Pramana 66:485-494, 2006.

[14] E. C. Titchmarcsh. The theory of functions. Oxford University Press, 1939.

[15] M. N. Tran, M. V. N. Murthy and R. K. Bhaduri. On the quantum density of states and partitioning an integer. Ann. Physics, 311:204-219, 2004.

[16] A. Vershik. Statistical mechanics of combinatorial partitions and their limit configurations. Funct. Anal. Appl. 30:90-105, 1996.

[17] E. T. Whittaker and G. N. Watson. A course of modern analysis. Cambridge University Press, 1996.

[18] Y. Yakubovich. Ergodicity of multiplicative statistics. J. Combin. Theory, A 119:1250-1279, 2012. 